\title{
Minimal Inclusive Sets in Special Classes of Games
}

by

Chris W. Sanchirico, Columbia University

November 1994 (August 1996)

Discussion Paper Series No. 9596-23

$d p 9596-23$ proges is 
MINIMAL INCLUSIVE SETS IN SPECIAL CLASSES OF GAMES

By Chris William Sanchirico ${ }^{1}$

November 1993

(This Draft: June 1994)

Note: This working paper is an expanded version of Section 4 of the published version of

“A Probabilistic Model of Learning in Games," forthcoming in Econometrica.

A companion paper, Sanchirico (1996), provides a probabilistic theory of learning in games with the convergence property that, almost surely, play will remain almost always (i.e., forever after some point) within one of the stage game's "minimal inclusive sets." This paper investigates the size of minimal inclusive sets in several classes of games, notably, those for which other learning processes have been shown to converge (in various manners weaker than convergence of actual play). These include certain supermodular games, congestion games, potential games, games with identical interests, and games with bandwagon effects. It is shown that in all these classes, if all of a game's pure equilibria are strict ( $a$ fortiori, if its payoffs are generic), then all of its minimal inclusive sets will be singletons consisting of Nash equilibria.

KEYWORDS: Rationalizability, Learning. 


\section{Introduction}

In recent years, much research has been directed toward formalizing the intuition that common knowledge of strategic intent may arise from repeated interaction. ${ }^{2}$ In this vein, Sanchirico (1996) provides a probabilistic theory of learning in games (in a repeated game framework) with the convergence property that, almost surely, play will remain almost always (i.e., forever after some point) within one of the stage game's "minimal inclusive sets" An "inclusive set" (DEFINITION 1; following Basu and Weibull ${ }^{4}(1991)$ ) is a rectangular subset of action profiles with the property that each player's factor includes all best responses to beliefs on the product of her opponents' factors. A minimal inclusive set (DEFINITION 2) is an inclusive set that contains no other inclusive set.

One of the two key assumptions that generate convergence in Sanchirico (1996) is a best response based noise assumption designed to reflect the indeterminacy of strategic interaction. One byproduct of this assumption is that convergence to any strict subset of a minimal inclusive set is impossible. According to the theory of learning in that paper, therefore, the potency of the learning approach to generate common knowledge of strategic intent varies across games according to the size of the game's minimal inclusive sets. The range of size of these sets is large. In some games, such as matching pennies, the only minimal inclusive set will be the entire set of (rationalizable ${ }^{5}$ ) profiles. In others, such as the battle of the sexes, all minimal inclusive sets will be pure strategy Nash equilibria.

This paper investigates the size of minimal inclusive sets in various classes of games. Special attention is paid to the several classes for which other learning processes have been shown to converge (in various manners weaker than convergence of actual play). These include, first, (certain) supermodular games (Krishna (1990)), congestion games (Rosenthal (1973)), potential games and games with identical interests (Monderer and Shapley (1993a and b)). In these the beliefs generated by fictitious play have been shown to converge to a mixed equilibrium of the stage game. Also considered are games with bandwagon effects (Kandori and 
Rob (1992)), in which the limit population distribution in the evolutionary model of Kandori and Rob (1992a) concentrates on a (particular) pure equilibrium. It is shown that in all such classes, if all of a game's pure equilibria are $\operatorname{strict}^{6}$ ( a fortiori, if its payoffs are generic ${ }^{7}$ ), then all of the game's minimal inclusive sets will be singletons consisting of strict equilibria.

The paper, therefore, accomplishes two tasks. In the first place, it shows that within the foregoing classes of games the probabilistic learning model in Sanchirico (1996) generates convergence of actual play to Nash equilibrium. But apart from its connection to Sanchirico (1996), the paper has interest in its own right in that it helps to tie together the growing menagerie of seemingly disparate classes of games for which positive convergence results have been obtained.

In broad outline, what these classes have in common are two important properties. The first is that all games within these classes have pure strategy equilibria. The second is that the properties that define these classes are "restrictable" to any one of the game's inclusive sets: that is, for any (rectangular) inclusive set, $I$, in any game $G$ with the property, the game $G_{I}$, with strategy space $I$ and payoffs equal to the restriction of $G$ 's payoffs to $I$, also has the property. (Supermodular games are not restrictable and we will have to modify the definition slightly to make them so.) This in turn implies that for each inclusive set $I$, the restricted game $G_{l}$ has a pure strategy equilibrium. By the definition "inclusive set," such an equilibrium must also be an equilibrium in $G$. If all pure equilibria in $\mathrm{G}$ are strict, each inclusive set will contain a strict equilibrium in $G$. But such strict equilibria are themselves inclusive sets. Hence, no minimal inclusive set can be other than a singleton consisting of a pure, strict equilibrium. This argument is formalized in THEOREM 1 (Template).

The work in this paper extends the research reported in Basu and Weibull (1991). That paper defines and establishes certain properties of inclusive sets and minimal inclusive sets. These are: $i$ ) the existence of minimal inclusive sets in a broad class of games (existence is trivial in the finite games that I consider here); ii) the "tightness" of minimal inclusive sets--the fact that the inclusive property holds with (set) equality for these sets; iii) the fact that every 
compact inclusive set contains the support of a Nash equilibrium; and $i v$ ) the fact that the set of rationalizable profiles is the largest "tight" inclusive set ${ }^{8}$. Basu and Weibull (1991) does not investigate the size of minimal inclusive sets in various classes of games. Nor does it discuss the application of such sets to the context of learning in games.

After presenting the general result discussed above in SECTION 2, I turn in SECTION 3 to the relationship between minimal inclusive sets and mixed equilibria. Then in SECTION 4, I take up supermodular games ((DEFINITION 6; Topkis (1978), Vives (1990), Milgrom and Roberts (1990), Milgrom and Shannon (1991)). Milgrom and Roberts (1991) have shown that if a supermodular game has a unique equilibrium, then any sequence consistent with "sophisticated learning" will converge to that equilibrium. Since supermodular games do not, in general, have unique equilibria (e.g., battle of the sexes), and since, when they do, such an equilibrium will be the game's only serially undominated strategy (Milgrom and Roberts (1990) Theorem 5 and Corollary), this convergence result does not directly address the question of whether learning models can show the formation of common knowledge of strategic intent, given common knowledge of rationality. Krishna (1991), however, has shown that in a particular subclass of supermodular games--those with completely ordered action spaces and "diminishing" increasing dif and only iferences--the sequence of beliefs generated by fictitious play ${ }^{10}$ (whose corresponding sequence of play will be consistent with sophisticated learning) will converge to one of the game's mixed equilibria. In SECTION 4, I show, inter alia, that in all supermodular games with generic payoffs ${ }^{11}$ in which each minimal inclusive set is a supermodular game in its own right (which will be the case if action spaces are completely ordered), all minimal inclusive sets will be singletons consisting of strict equilibria (COROLLARY 2).

More recently, Monderer and Shapley (1993a and b) have investigated the properties of fictitious play in "potential games," (which include Rosenthal's (1973) "congestion games") and games "with identical interests" (DEFINITIONS 9 and 14, respectively). These authors show in Monderer and Shapley (1993a) that in games with identical interests, the beliefs generated by fictitious play converge to a mixed equilibrium of the game. In Monderer and Shapley (1993b), 
it is noted that the class of games with identical interests includes the class of "weighted" potential games. In SECTION 5, I show that in all "ordinal" potential games (a broader class than weighted potential games) as well as all games with identical interests, all minimal inclusive sets will be singletons consisting of strict equilibria (subject again to the genericity of payoffs).

Lastly, Kandori and Rob (1992) identify a class of symmetric two player games in which their evolutionary model (Kandori and Rob (1992a) following Foster and Young (1990) and Kandori, Malaith and Rob (1992a)) will converge in the particular sense they specify (see discussion in SECTION 6, infra). These are games with "bandwagon effects," wherein the benefit of switching to a given action is greatest when all others are using that action. In SECTION 6, I show that in all such games (no additional genericity requirement is necessary), all minimal inclusive sets are singletons consisting of pure equilibria.

\section{Definitions}

Fix a game $G=\left(A_{1}, \ldots, A_{n} ; \pi_{1}, \ldots, \pi_{n}\right)$, where for each player $i, A_{i}$ is $i$ 's finite set of actions and $\pi_{i}: A_{1} \times \mathrm{K} \times A_{n} \rightarrow \Re$ is $i$ 's payoff function. Let $\Gamma$ denote the set of all such games. For any subset $E_{-i} \subseteq A_{-i}$ of opponent action profiles, ${ }^{13}$ let $\Delta\left(E_{-i}\right)$ denote the set of all probability measures $\psi_{-i}$ on $A_{-i}$ (with the power set as $\sigma$-algebra) with $\psi_{-i}\left(E_{-i}\right)=1$. Extend $\pi_{i}$ to an expected payoff function $u_{i}: A_{i} \times \Delta\left(A_{-i}\right) \rightarrow \mathfrak{R}$ in the usual manner. The set of (stage game) best responses for player $i$ to the belief $\psi_{-i} \in \Delta\left(A_{-i}\right)$ is, as usual,

$$
b_{i}\left(\psi_{-i}\right) \equiv\left\{a_{i} \in A_{i} \mid u_{i}\left(a_{i}, \psi_{-i}\right) \geq u_{i}\left(\$, \psi_{-i}\right), \forall \$ \in A_{i}\right\} .
$$

The set of (stage game) best responses for player $i$ to beliefs on any subset $E_{-i} \subseteq A_{-i}$ is

$$
b_{i} \Delta \Delta\left(E_{-i}\right) \equiv \bigcup_{\psi, \in \Delta(E,)} b_{i}\left(\psi_{-i}\right)
$$

Based on these definitions we may define a type of subset of action profiles that includes all best responses to itself. 
Definition 1 [Inclusive and Exact Sets; Basu and Weibull (1990)]: A non empty subset of action profiles $E=E_{1} \times \mathrm{K} \times E_{n} \subseteq A$ is (best response) inclusive, if and only if $b_{i} \Delta \Delta\left(E_{-i}\right) \subseteq E_{i}$, all i.. The set $E$ is exact if $b_{i} \Delta \Delta\left(E_{-i}\right)=E_{i}$, all $i$.

EXAMPLE 1 [Some Familiar Inclusive Sets]: In any given game $G$, the whole set of profiles $A$ is inclusive as is the set of rationalizable profiles (Bernheim (1984) and Pearce (1984)). Any strict Nash equilibrium, regarded as a singleton, is inclusive, but Nash equilibria that are not strict are not inclusive. GAME 1, infra, has three inclusive sets, the whole set of profiles and the two strict equilibria taken as singletons.

REMARK 1 ["Exclusive" Sets]: Inclusive sets should be distinguished from what we might, by symmetry, call "exclusive sets:" that is, sets with the reverse inclusion $E_{i} \subseteq b_{i} \circ \Delta\left(E_{-i}\right)$, all $i$. Such subsets "exclude" all profiles that are "never best response" on $E$. An exact set is both inclusive and exclusive. All equilibria, whether or not they are strict, are exclusive sets when regarded as singletons. Moreover, the set of rationalizable profiles is the largest exclusive set (Bernheim (1984) and Pearce (1984)) and hence the largest exact set as well. The whole set of profiles may not be exact or exclusive.

There is an interesting duality between inclusive and exclusive sets, as laid out in Sanchirico (1994). While inclusive sets are closed under intersection, exclusive sets are closed under (factorwise) union. Hence, for every subset of profiles there is a smallest inclusive set containing that subset as well as a largest exclusive set contained in that set. Lastly, while this smallest containing inclusive set may be found by a process of iterated addition of best responses (starting from the contained subset), the largest contained exclusive set may be found by a process of iterated elimination of never best responses.

DEFINITION 2 [Minimal Inclusive Sets]: An inclusive set $I$ is minimal if it contains no other inclusive set $I^{\prime} \neq I$. 
EXAMPLE 2 [Examples of Minimal Inclusive Sets; Cooperative versus Competitive]:

One's first guess might be that minimal inclusive sets are small in games with much common interest and large in games that exhibit conflict. The only minimal inclusive sets in GAME 1 , for instance, are the two pure equilibria, while the entire set of profiles is minimal in the familiar zero-sum game, matching pennies. But the intuition is generally false. Some games that seem to exhibit a large degree of "common interest" have large minimal inclusive sets. In GAME 2, for instance, the entire set of profiles is minimal inclusive, though the two players have a strong mutual interest in playing $(\mathrm{P}, P)$. Inversely, games of "pure conflict" may have small minimal inclusive sets. GAME 3, "Augmented Matching Pennies" is a zero sum game (in which all profiles are rationalizable) whose only minimal inclusive set is its unique pure equilibrium, $(\mathrm{B}, R)$.

After the requisite definitions, I provide the fairly straightforward argument that serves as a "template" for the succeeding results on the specific classes of games considered in the following sections.

Definition 3 [Restriction of Game to a Subset of Action Profiles]: Let $G=(A, \pi)$ be a finite game. Let $E$ be a rectangular subset of $A$. The restriction of $G$ to $E$, denoted $G_{E}$ is the finite normal form with strategy sets $E_{i}$ and payoffs $\pi_{i} \mid E$.

DEFINITION 4 [Restrictability to Inclusive Sets]: Let $\mathrm{P}$ be a property defined on games in $\Gamma$. The property $\mathrm{P}$ is restrictable to inclusive sets, if for all games $\mathrm{G}$ with the property and all inclusive sets $I$ in $G$, the restriction $G_{l}$ of $G$ to I also has the property. 
EXAMPLE 3: The property: "has no more than one (pure strategy ${ }^{14}$ ) equilibrium" is restrictable to inclusive sets, since all equilibria in the restriction of $G$ to an inclusive set are equilibria in $G$ as well.

This property is not, however restrictable to general subsets of profiles. For example, GAME 3 has one pure equilibrium, yet in the restriction of GAME 3 to $\{M, B\} \times\{M, R\}$, both diagonal profiles are pure equilibria.

The property "has no less than one (pure) equilibrium" is not restrictable even to inclusive sets. Again considering GAME 3, if the " $1 / 2$ "'s and " $-1 / 2$ "" are replaced by zeros, then the whole game would have one pure equilibrium, $(B, R)$, but the restriction of the game to $\{T, M\} \times\{L, M\}$, now an inclusive set, has no pure equilibrium.

REMARK 2: The property $P$ can, of course, be the conjunction or disjunction of several properties.

THEOREM 1 [Template]: If all games with the property $P$ have (pure strategy) Nash equilibria, and $P$ is restrictable to inclusive sets, then in all games with $P$ for which all pure equilibria are strict, all minimal inclusive sets are singletons consisting of strict equilibria.

Proof: Let $G$ have property $P$ as well as the property, call it $P^{\prime}$, that all its (pure) equilibria are strict. Consider any minimal inclusive set $I$ for $G$. Since both $P$ and $P^{\prime}$ are restrictable to inclusive sets, the restriction of $G$ to $I, G_{I}$, has a strict equilibrium, $a$. Since $I$ is inclusive, $a$ must also be a strict equilibrium for $G$. But all strict equilibria, as singletons, are inclusive sets. Therefore, since $I$ is minimal, it must consist solely of this equilibrium, $a$.

REMARK 3: It is clear from the proof that the property in the statement of the theorem need only be restrictable to minimal inclusive sets. 


\section{Minimal Inclusive Sets and Mixed Equilibria}

As a first application of the general result, I consider the class of games whose "mixed extensions" have the property that the support of every equilibrium contains a pure equilibrium. This class includes games whose mixed extensions have only pure equilibria. But, as Gul, Pearce and Stacchetti (1990) have shown, all generic games with more than one equilibrium have a non degenerate mixed equilibrium. Hence, this latter class is essentially the same as the class of games whose mixed extensions have a unique equilibrium in pure strategies. This includes, for example, the zero sum GAME 3, supra.

Along the way to the main result, I consider the general relationship between mixed equilibria (in the mixed extension) and minimal inclusive sets.

Definition 5 [Mixed Extension of Finite Game]: The mixed extension of game $\mathrm{G}$ is the (non finite) game $G^{\prime}=\left(A^{\prime}, \pi^{\prime}\right)$ with action spaces $A_{i}^{\prime}=\Delta\left(A_{i}\right)$ and payoff functions $\pi_{i}^{\prime}$ defined from $\pi_{i}$ via the usual expected utility calculation. The mixed extension of a rectangular subset of action profiles $E \subseteq A$ in the finite game $G$ is the subset $\Delta\left(E_{1}\right) \times \ldots \Delta\left(E_{n}\right)$ of $A^{\prime}$.

REMARK 4 [Definition of Inclusive Sets for Mixed Extension]: The definition of "inclusive set," "exact set" and "minimal inclusive set" are readily extended to the mixed extension.

Mixed equilibria and minimal inclusive sets are obviously related by the fact that if a mixed extension of a minimal inclusive set contains a (mixed) equilibrium of (the mixed extension of $G$, then the support of that equilibrium must of course be contained in that minimal inclusive set. However, two complications make the connection between the support of mixed equilibria and minimal inclusive sets less than straightforward. 
EXAMPLE 4 [Mixed Extension of Minimal Inclusive Set in $G$ is Not Necessarily Minimal Inclusive in Mixed Extension of $G$ ]: Consider any subset $E$ of action profiles in the finite game $G$. Consider, next, for any player $i$, the support of the set of best responses to the mixed extension of $E, E^{\prime}$, in the mixed extension of $G$ which we might denote as supp $b_{i}^{\prime} \circ \Delta\left(E_{-i}^{\prime}\right)$. Clearly, this is equal to the set of best responses to $E_{-i}$ in the finite game, $b_{i} \circ \Delta\left(E_{-i}\right)$. However, the mixed extension of $b_{i} \circ \Delta\left(E_{-i}\right)$ may strictly contain $b_{i}^{\prime} \circ \Delta\left(E_{-i}^{\prime}\right)$. For example, two (pure) strategies may be in $b_{i} \circ \Delta\left(E_{-i}\right)$ because they are best responses to two different opponent profiles in $E_{-i}$. There is then no reason to believe that any given mixture of these two profiles is best response to any belief on $E_{-i}$.

From this fact arises the possibility that the mixed extension of a minimal inclusive set may not necessarily be minimal inclusive. Consider, for example, the zero sum game, GAME 3 (Augmented Matching Pennies). Modify the game slightly by replacing the " 0 " payoff in the southeastern most cell with a " 1 ." Then the entire set of profiles is minimal inclusive. But Row, for example, would never want to $\operatorname{mix} T$ and $M$ with equal weight, since $B$ dominates this mixed strategy. Therefore, the mixed extension of the entire set of profiles cannot be minimal inclusive.

EXAMPLE 5: As noted an inclusive set will contain the support of any mixed equilibrium in its mixed extension. However, the mixed extension of a minimal inclusive set may contain more than one mixed equilibrium. Non-generic examples are trivial to construct. For a robust example, consider GAME 4. It is easy to check that the game has no pure equilibria and the only inclusive set is the entire set of profiles. Hence, the game has one minimal inclusive set. Yet the mixed profiles $\left(\frac{1}{2} 1+\frac{1}{2} 2, \frac{1}{2} 1+\frac{1}{2} 2\right)$ and $\left(\frac{1}{2} 3+\frac{1}{2} 4, \frac{1}{2} 3+\frac{1}{2} 4\right)$ are both equilibria.

Nevertheless, it is possible to make several interesting statements about the relationship between mixed equilibria and minimal inclusive sets. 
LEMma 1 [Fewer Minimal Inclusives than Mixed Equilibria; Based in Part on Basu and Weibull (1990)]: There are no more minimal inclusive sets in $G$ than there are mixed equilibria in G's mixed extension.

Proof: First, I work with the mixed extension and establish a one to one map from the mixed extension's minimal inclusive sets to its mixed equilibria. Next, I establish a one to one map from the minimal inclusive sets of the finite game to the minimal inclusive sets of the mixed extension. The existence of the composite one to one map proves the result.

Consider the mixed extension $G^{\prime}$, of $G$. As noted by Basu and Weibull (1990), each compact inclusive set in $G^{\prime}$ contains, by the usual fixed point argument, a Nash equilibrium of the mixed extension $G^{\prime}$. Basu and Weibull (1990) also show that every minimal inclusive set $I^{\prime}$ in $G^{\prime}$ is exact. From this it follows that every minimal inclusive set in $G^{\prime}$ is compact and therefore contains an equilibrium. Now minimal inclusive sets must be disjoint, since inclusive sets are closed under intersection. Hence, no equilibrium for $G^{\prime}$ is contained in more than one minimal inclusive set in $G^{\prime}$. Therefore, there are no more minimal inclusive sets in the mixed extension $G^{\prime}$ than there are mixed equilibria therein.

To show that this implies that the same holds for the finite game $G$, it suffices to show that the set of minimal inclusive sets in $G$ can be mapped one to one into the set of minimal inclusive sets in $G^{\prime}$. Take any minimal inclusive set $I$ in the finite game $G$. Consider the mixed extension, $I$ ' of $I$. While $I$ ' is not necessarily minimal inclusive, it is easy to show that it must at least be inclusive. Since it is also compact, it must contain a minimal inclusive set $I^{\prime}$ in $G^{\prime}$, as shown by Basu and Weibull (1991). In this manner we associate each minimal inclusive set in G with one minimal inclusive set in $G^{\prime}$. Since minimal inclusive sets in $G$ are disjoint, it follows that this association must be one to one.

It is trivial that minimal inclusive sets exist in all finite games. One simply starts with the whole set of profiles (an inclusive set) and then proceeds to smaller and smaller inclusive sets 
until the process stops. Hence, as a solution concept, minimal inclusive sets do not have the existence problem of Nash equilibrium, which originally inspired resort to concept of a mixed strategy. (Of course, minimal inclusive sets need not be singletons.) LEMMA 1 may be interpreted as showing that minimal inclusive sets have no worse a multiplicity problem than mixed equilibria.

The main result in this section follows more easily than LEMMA 1.

COROLlaRY 1 [Sufficient Condition for Singleton Minimal Inclusive Sets]: If, in the mixed extension of $G$, the support of every mixed equilibrium contains a pure equilibrium and all pure equilibria are strict, then these pure equilibria, as singletons, are G's only minimal inclusive sets.

Proof [Using THEOREM 1 (Template)]: The assumption implies the existence of a pure equilibrium in $G$ 's mixed extension and any pure equilibrium for $G$ 's mixed extension is an equilibrium for $G$ itself. Hence, all that remains for application of THEOREM 1 (Template) is to show that the property, "the support of each of the mixed extension's mixed equilibria contains a pure equilibrium," is restrictable to inclusive sets. But because of the definition of inclusive set, any mixed equilibrium for the mixed extension of the restriction of $G$ to an inclusive set $I$--call this game $G_{I}{ }^{\prime}$--is as well an equilibrium for the mixed extension $G^{\prime}$ of $G$. Moreover, if a profile is not a pure equilibrium in $G_{I}{ }^{\prime}$, then it is certainly not a pure equilibrium in $G^{\prime}$. Hence, if $G_{I}^{\prime}$ contained a mixed equilibrium with no pure equilibrium in its support, the same would be true of $G^{\prime}$, contradicting our assumption.

REMARK 5 [Strict Pure Equilibria weaker than Generic Payoffs]: The property that all pure equilibria are strict is generic in the space of all finite games. (See, e.g. Fudenberg and Tirole (1991), Chapter 12) 


\section{Supermodular games}

As mentioned in the introduction, Krishna (1991) shows that in the subclass of supermodular games in which strategy spaces are completely ordered and there are "diminishing increasing differences," the empirical frequency of play converges to one of the games (possibly) mixed equilibria. The subclass of supermodular games that I consider here is not logically related to Krishna's. The class includes some games in which strategy spaces are not completely ordered, but only games in which all pure equilibria are strict. ${ }^{15}$

We start by reminding the reader of Milgrom and Roberts' (1990) definition of supermodular games. For a full definition, please see that paper.

DEFINITION 6 [Supermodular Finite Games; Milgrom and Roberts (1990)]: A finite game G whose action sets $A_{i}$ are partially ordered ${ }^{16}$ is supermodular, if and only if ${ }^{17}$ :

i) $\left(A_{i}, \geq_{i}\right)$ is a complete lattice ${ }^{18}$, all $\mathrm{i}$.

ii) $\pi_{i}$ is supermodular ${ }^{19}$ in $a_{i}$

iii) $\pi_{i}$ has increasing differences ${ }^{20}$ in $a_{i}$ and $a_{-i}$.

REMARK 6 [Discussion of Supermodular Games]: If strategy spaces are completely ordered, then they are complete lattices. Moreover, the requirement that $\pi_{i}$ be supermodular becomes vacuous, and the class of supermodular games collapses into those with "strategic complementarities," as enforced by the requirement of "increasing differences:" that is, games in which players best responses increase (in the specified complete ordering) in the strategies of their opponents. In the broader class of supermodular games the requirement that players' strategy spaces are completely ordered is relaxed to the requirement that they be complete lattices. Added is the condition that $\pi_{i}$ be supermodular in $a_{i}$ on this complete lattice -- roughly, that the dimensions of players' strategy spaces are complementary "inputs" in producing payoffs. (See Milgrom and Roberts (1990) for more discussion.) 
The next step is to define the subclass of supermodular games that are restrictable to inclusive sets. The only part of the definition of supermodularity that poses a problem is the requirement that action spaces be complete lattices. A subset of a complete lattice is not necessarily a complete lattice in its own right. (The subset $\{(0,1),(1,0)\}$ of the complete lattice $\{(0,0),(0,1),(1,0),(1,1)\}$ with the (restriction of the) usual ordering on $\mathfrak{R}^{2}$ is not a complete lattice since, e.g., it fails to contain an upper bound for itself). Hence, the following definition:

DEFINITION 7 [Complete Lattice in Own Right]: Let $(S, \geq)$ be a partially ordered set. A subset $\mathrm{T}$ of $\mathrm{S}$ is a complete lattice in its own right, if $\mathrm{T}$ coupled with the restriction of $\geq$ to $\mathrm{T}$ (i.e. $\geq \cap[T \times T])$ is a complete lattice.

LEMMA 2 [Restriction of Supermodular Game]: Let $G$ be a finite supermodular game. Let $E$ be a rectangular subset of profiles in $A$. If each $E_{i}$ is a complete lattice in its own right, then the restriction of $G$ to $E$ is a supermodular game.

Proof: Each $E_{i}$ is a complete lattice by assumption and the other requirements in the definition of supermodular games clearly survive restriction to E.

REMARK 7: A stronger assumption for this lemma would be that $A_{i}$ is a complete sublattice of $\left(A_{i}, \leq_{i}\right)$. (See Milgrom and Roberts (1990)).

DEFINITION 8 [Restrictable Supermodular Games]: In light of LEMMA 2, a supermodular game is restrictable, if for all inclusive sets $\mathrm{I}$, each $I_{i}$, is a complete lattice in its own right. 
Milgrom and Roberts (1990) THEOREM 5 shows that all supermodular games have pure equilibria. In particular, such games admit both a largest and a smallest "serially undominated strategy," and these will be pure equilibria. It then follows immediately from THEOREM 1 (Template):

COROLlaRY 2 [To Milgrom and Roberts (1990), Theorem 5]: If $G$ is a finite, restrictable supermodular game all of whose pure equilibria are strict, then all minimal inclusive sets in $G$ are singletons consisting of strict equilibria.

REMARK 8: Actually, one can prove a bit more, namely: every minimal inclusive set that is a complete lattice in its own right is a singleton consisting of a pure equilibrium. ${ }^{21}$

Since any subset of a completely ordered set is also completely ordered, we obtain:

COROLlaRY 3 [Games with Completely Ordered Action Spaces]: If $G$ is a finite supermodular game with completely ordered action spaces and all of $G$ 's pure equilibria are strict, then all of $G$ 's minimal inclusive sets are singletons consisting of strict equilibria.

EXAMPLE 6 [Supermodular Games whose Minimal Inclusive Sets are not Complete Lattices in Their Own Right]: In the full class of supermodular games, even those with generic payoffs, minimal inclusive sets can be quite large. Consider a two player game in which players choose strategies along two dimensions, each dimension containing two choices. Let the choices for both players be the pairs $\{a=(0,1), b=(0,1), c=(0,0), d=(1,1)\}$, and endow this set with usual product ordering on $\mathfrak{R}^{2}$, so that, for example, $\mathrm{d}$ is the largest element, $\mathrm{c}$, the smallest and $\mathrm{a}$ and $\mathrm{b}$ are unrelated. Then both player's strategy spaces are complete lattices.

Assign payoffs to the players as in GAME 5. (Zeros are not shown). Then payoffs are supermodular for both players: fixing any column, for instance, we see that the sum of payoffs to 
Row for $\mathrm{a}$ and $\mathrm{b}$ are never greater than the sum of her payoffs for $\mathrm{c}$ and $\mathrm{d}$. In addition, both players' payoffs have increasing dif and only iferences: the benefit to Row (perhaps negative) of increasing his strategy from a to $d$, for instance, is no less when Column plays $d$ then when column plays $\mathrm{a}, \mathrm{b}$ or $\mathrm{c}$.

Therefore, the game is supermodular. However, the subset of profiles $\{a, b\} \times\{a, b\}$ is minimal inclusive. Moreover, the example is robust to changes in payoffs.

\section{Potential Games, Congestion Games and Games with Identical Interests}

Ordinal, weighted and exact potential games as well as games with identical interests were introduced to the literature on learning in two recent papers, Monderer and Shapley (1993a and $b$ ). The former paper proves a convergence result for fictitious play in games with identical interests. The latter defines the three nested types of potential games, shows that the class of games with identical interests includes the middle type, weighted potential games, and proves the equivalence of the smallest class, exact potential games to Rosenthal's (1973) "congestion games." Congestion games model situations wherein each of a group of players must travel from one node to another on a graph, with different starting points and destinations for each. The players choose which path to take and the payoffs to each driver depend on the number of other players that also use the branches along the path she chooses. Monderer and Shapley do not provide an interpretation for the broader classes of ordinal and weighted potential games, preferring to view the classes solely as helpful mathematical classifications. I first consider potential games, providing a result for the broadest of the three classes. Then I move to games with identical interests.

\subsection{Potential Games}

First some definitions. 
Definition 9 [Ordinal Potential Function and Game; Monderer and Shapley (1993b)]: A function $P: A \rightarrow \mathfrak{R}$ is an ordinal potential for the finite normal form game $G=(A, \pi)$, if for every player $\mathrm{i}$ and every $a_{-i} \in A_{-i}$

$\forall a_{i}, \alpha \% \in A_{i} \quad \pi_{i}\left(a_{i}, a_{-i}\right)-\pi_{i}\left(\delta / a_{a-i}\right)>0$, iff $P\left(a_{i}, a_{-i}\right)-P\left(\alpha a_{-i}\right)>0$

The game $\mathrm{G}$ is an ordinal potential game if it admits an ordinal potential function.

The authors define two (nested) subclasses of such games:

Definition 10 [Weighted Potential Function and Game; Monderer and Shapley (1993b)]: Let $w=\left(w_{1}, \ldots, w_{n}\right) \in \mathfrak{R}_{++}^{n}$ be a vector of positive weights. A function $P: A \rightarrow \Re$ is a $w$-potential for the finite normal form game $G=(A, \pi)$, if for every player i and every $a_{-i} \in A_{-i}$

$\forall a_{i}, \not \% \in A_{i} \quad \pi_{i}\left(a_{i}, a_{-i}\right)-\pi_{i}\left(\not / a, a_{-i}\right)=w_{i}\left(P\left(a_{i}, a_{-i}\right)-P\left(\& / a, a_{-i}\right)\right)$

The game $\mathrm{G}$ is a w-potential game if it admits a w-potential function. When not interested in the identity of the weights, we say that such $P$ is a weighted potential function and such $G$, a weighted potential game.

As noted by Monderer and Shapley (1993b), the class of weighted potential games is a subclass of the class of games with identical interests, considered below.

Definition 11 [Exact Potential Game; Monderer and Shapley (1993b)]: A function $P: A \rightarrow \Re$ is an (exact) potential for the finite normal form game $G=(A, \pi)$, if it is a wpotential for $\mathrm{G}$ with weight $w_{i}=1$, for all $\mathrm{i}$. The game $\mathrm{G}$ is an (exact) potential game it is admits an exact potential function. 
As noted by Monderer and Shapley (1993b) COROLLARY 2.2, all finite ordinal potential games have (pure) equilibria. In particular, a profile that maximizes the potential function will be an equilibrium. Moreover, it is clear from the definition of ordinal potential games that the property defining such games is restrictable to inclusive sets (indeed to all subsets). Then from THEOREM 1 (Template) all ordinal potential games (hence all weighted and exact potential games) whose pure equilibria are strict have singleton minimal inclusive sets:

Corollary 3 [Minimal Inclusive Sets are Singletons in Ordinal Potential Games]: If $G$ is a finite ordinal potential game in which all pure equilibria are strict, then every minimal inclusive set in $G$ is a singleton consisting of a strict Nash equilibrium.

\subsection{Games with Identical Interests}

A game with identical interests is simply one whose "best response regions" correspond to those of a game with identical payoffs. Formally,

DEFINITION 12 [Best Response Equivalence in Mixed Strategies; Monderer and Shapley (1993a)]: The game $G=(A, \pi)$ is best response equivalent in mixed strategies to the game $G^{\prime}=\left(A^{\prime}, \pi^{\prime}\right)$, if $A=A^{\prime}$ and for every player i and every belief/opponent mixed strategy, $\psi_{-i} \in \Delta\left(A_{-i}\right), b_{i}\left(\psi_{-i}\right)=b_{i}^{\prime}\left(\psi_{-i}\right)$, where, naturally, $b_{i}^{\prime}$ denotes i's best response correspondence in $G^{\prime}$

DEFINITION 13 [Fully Symmetric]: A game $G=(A, \pi)$ is fully symmetric, if $\forall i \neq j, A_{i}=A_{j}$ and $\pi_{i}=\pi_{j}$. 
DEFINITION 14 [Games with Identical Interests]: The normal form $\mathrm{G}$ is a game with identical interests, if it is best response equivalent in mixed strategies to some fully symmetric game.

As noted by Monderer and Shapley (1993a), p.9, all games with identical interest have (pure) equilibria. (Use the payoff function in the equivalent fully symmetric game as an ordinal potential function.) Moreover, it is clear from the definition of inclusive sets, that if $G$ is best response equivalent in mixed strategies to the fully symmetric $\%$, then $G_{I}$ is best response equivalent in mixed strategies to $\mathscr{G}$. Hence, the property defining games with identical interests is restrictable to inclusive sets (though it is not restrictable to general subsets). Once again applying THEOREM 1 yields:

COROLlaRy 4 [All Minimal Inclusives are Singletons in Games with Identical Interests]: If $G$ is a game with identical interests, and all its pure equilibria are strict, then all its minimal inclusive sets are singletons consisting of strict Nash equilibria.

\section{Games with Bandwagon Effects}

Kandori, Malaith and Rob (1992) and Kandori and Rob (1992a), following Foster and Young (1990), consider an evolutionary model in which a fixed finite population of players are repeatedly and randomly paired to play a given finite two player stage game. The process takes the form of a Markov chain whose state vector is the number of players in the population using each (pure) strategy in the stage game. Transition probabilities are determined by two factors. The first is myopic best response play with "inertia." Players "tend" to take the same action this period as they did last period. But there is always some fixed probability that each member of the population will instead choose an action that is best response to the population distribution of actions taken last period. The second factor is "mutation." In each period there is some small 
probability that any given member of population switches to any given action, whether or not it is best response to last period's population distribution. For each set of parameters, the resulting Markov chain has a unique stationary distribution. Taking the limit of this distribution as the probability of mutation goes to zero gives the "limit distribution." The "long run states" are those which receive positive probability thereunder.

For general games this Markov process will not necessarily converge in the sense that the limit distribution will not put unit weight on one state. However, in Kandori and Rob (1992b), the authors identify a class of two player symmetric games wherein the only long run state is one in which everyone in the population plays some Nash equilibrium. These are games with the "marginal bandwagon property." Such games are meant to reflect situations in which "the value of a technology to one user depends on how many others are adopting it." The helpful example carried throughout the paper concerns the choice of personal computer among individual faculty in an economics department. A given professor is better off choosing to order a Macintosh, for example, if all his colleagues have done the same. The formal bandwagon property is captured in the following definition. Since definition is for symmetric two player games only, I simplify notation by dropping the player subscripts and writing $\pi(a, \$)$ for the payoff of the player playing the first strategy, $a$, in the pair:

Definition 15 [Marginal Bandwagon Property; Kandori and Rob (1992b)]: A two player, finite and symmetric game $\mathrm{G}$ has the marginal bandwagon property, if and only if for all distinct actions $a, a^{\prime}, a^{\prime \prime} \in A$,

$$
\pi(a, a)-\pi\left(a^{\prime}, a\right)>\pi\left(a, a^{\prime \prime}\right)-\pi\left(a^{\prime}, a^{\prime \prime}\right)
$$

REMARK 9 [Comparison with Increasing Differences and Supermodular Games]: Contrast the marginal bandwagon property with the property of increasing differences used in defining supermodular games. Increasing differences says that, given the ordering on strategy spaces, the 
"larger" your opponent's strategy, the greater the benefit of "increasing" your own strategy. The marginal bandwagon property says that the benefit of switching to a given strategy (whether that be an increase or a decrease) is (strictly) greatest when your opponents are using that strategy as well. A two player, three strategy game with $(1,1)$ on the main diagonal and $(0,0)$ elsewhere has the marginal bandwagon property but, one can show that it can not exhibit increasing dif and only iferences for any pair of partial orderings (which are, by definition, antisymmetric) on the strategy spaces.

Mathematically, the marginal bandwagon property has the very strong implication that if an action $a^{\prime}$ "beats" $a$ against $a$ itself $\left(\pi(a, a)-\pi\left(a^{\prime}, a\right)<0\right)$, then it beats $a$ against every other (pure) action as well, since then $\pi\left(a, a^{\prime \prime}\right)-\pi\left(a^{\prime}, a^{\prime \prime}\right)<0$ a and action $a^{\prime \prime}$ is chosen independently of $a$ and $a^{\prime}$. In short, if an action beats $a$ against itself, then it strictly dominates $a$. The contrapositive of this assertion is that if $a$ is undominated, then it must be a symmetric (pure strategy) Nash equilibrium. ${ }^{22}$ (Indeed, it will be strict.) Since every game contains some undominated strategy, we have that every game with the marginal bandwagon property contains a pure and strict equilibrium. Moreover, the marginal bandwagon property is, on its face, restrictable to inclusive sets (indeed to all sets). Therefore, THEOREM 1 (Template) is again applicable. More formally,

Corollary 5 [Generic Games with Marginal Bandwagon Property have All Singleton Minimal Inclusive Sets]: If $G$ has the marginal bandwagon property (and is therefore symmetric and two player), then all minimal inclusive sets in $G$ are singletons consisting of strict Nash equilibria.

Proof: First I show that every game with bandwagon effects contains a strict equilibrium. Take any undominated action $a$. For all strategies $a^{\prime}$ there exists a third strategy $a^{\prime \prime}$ s.t. $\pi\left(a, a^{\prime \prime}\right)-\pi\left(a^{\prime}, a^{\prime \prime}\right) \geq 0$. But by the marginal bandwagon property, 
$\pi(a, a)-\pi\left(a^{\prime}, a\right)>\pi\left(a, a^{\prime \prime}\right)-\pi\left(a^{\prime}, a^{\prime \prime}\right)$. Hence, $\pi(a, a)-\pi\left(a^{\prime}, a\right)>0$. The action $a^{\prime}$ having been chosen arbitrarily, and $\mathrm{G}$ being symmetric, $(a, a)$ must be a strict equilibrium.

Since it is clear that the marginal bandwagon property is restrictable to inclusive sets, the result follows the same reasoning as in THEOREM 1.

REMARK 10: There is no need for the additional requirement that all pure equilibria be strict in COROLLARY 5 because of the strict inequality in the definition of the marginal bandwagon property. 


\section{REFERENCES}

Basu, K. and J. W. Weibull (1991), "Strategy Subsets Closed Under Rational Behavior," Economics Letters, 36, 141-146.

Bernheim, B. D. (1984), "Rationalizable Strategic Behavior," Econometrica, 52:1007-1028.

Brandenberger, A. and E. Dekel (1987), "Rationalizability and Correlated Equilibrium," Econometrica, 55:1391-1402

Brown, G. W. (1951), "Iterated Solution of Games by Fictitious Play," Activity Analysis of Production and Allocation, John Wiley and Sons, pp 374-376

Cournot, A. (1838), Recherches sur les Principes Mathematiques de la Theorie des Richesses, English edition: Researches into the Mathematical Principles of the Theory of Wealth, ed. N. Bacon (Macmillan, 1897)

Crawford, V. (1992) “Adaptive Dynamics in Coordination Games," mimeo, University of California at San Diego.

Foster, D and H. P. Young, (1990)"Stochastic Evolutionary Game Dynamics," Theory of Population Biology, 38: 219-232.

Fudenberg, D. and D. Kreps, (1988), “A Theory of Learning, Experimentation and Equilibrium in Games," mimeo, Massachusetts Institute of Technology, Stanford Graduate School of Business. (1992), "Learning Mixed Equilibria," mimeo, Massachusetts Institute of Technology, Graduate School of Business, Stanford.

Fudenberg, D. and D. Levine, (1993a), "Self-Confirming Equilibrium," Econometrica, 61: 523545. 
(1993a), "Steady State Learning and Nash Equilibrium," Econometrica, 61: 547573.

Fudenberg, D. and J. Tirole (1991), Game Theory, Cambridge Massachusetts, MIT Press.

Gilboa, I. and D. Samet (1991), “Absorbent Stable Sets,” mimeo, J. L. Kellogg Graduate School of Management, Northwestern University.

Gul, F. (1991), "Rationality and Coherent Theories of Strategic Behavior," mimeo, Stanford University.

Gul, F., D. Pearce, E. Stacchetti (1990), "A Bound on the Proportion of Pure Strategy Equilibria in Generic Games," Cowles Foundation Discussion Paper No. 981, Yale University.

Jordan, J. (1991), "Bayesian Learning in Normal Form Games," Games and Economic Behavior, 3:60-81.

(1991b) "Bayesian Learning in Repeated Games," mimeo, University of Minnesota.

Kalai, E. and E. Lehrer (1990a), "Bayesian Learning and Nash Equilibrium," Northwestern University.

(1990b), "Merging Economic Forecasts," Northwestern University. (1992), "Bounded Learning Leads to Correlated Equilibrium," Northwestern University. (1993a), "Rational Learning Leads to Nash Equilibrium," Econometrica, 61: 1019-1045. (1993b), "Subjective Equilibrium in Repeated Games," Econometrica, 61: 12311240. (1993c), "Weak and Strong Merging of Opinion," Journal of Mathematical Economics, (forthcoming) 
Kandori, M, G. Malaith and R. Rob, (1992), "Learning, Mutation and Long Run Equilibria in Games," Econometrica, (forthcoming).

Kandori, M. and R. Rob, (1992a), "Evolution of Equilibria in the Long Run: A General Theory and Applications," CARESS Working Paper No. 92-06, University of Pennsylvania. (1992b), "Bandwagon Effects and Long Run Technological Choice," mimeo, Faculty of Economics, University of Tokyo and Department of Economics, University of Pennsylvania.

Krishna, V. (1991), "Learning in Games with Strategic Complementarities," mimeo, Harvard Business School.

Li Calzi, M. (1992), “Fictitious Play by Similarities,” mimeo, Stanford University Matsui, (1993), "Evolution and Rationalizability," CARESS Working Paper No. 93-19, University of Pennsylvania

Maynard Smith, J., (1982), Evolution and the Theory of Games, Cambridge, Cambridge University Press.

Maynard Smith, J. and G. Price, (1973) "The Logic of Animal Conflict," Nature, 246: 15-18

Milgrom, P. and J. Roberts (1990), "Rationalizability, Learning, and Equilibrium in Games with Strategic Complementarities," Econometrica, 58: 1255-1277 (1991), "Adaptive and Sophisticated Learning in Normal Form Games," Games and Economic Behavior, 3: 82-100

Milgrom, P. and C. Shannon (1991), "Monotone Comparative Statics," Technical Report No. 11, Stanford Institute for Theoretical Economics, Stanford University. 
Monderer, D. and Shapley, L. S.(1993a), "Fictitious Play Property for Games with Identical Interests," mimeo, Faculty of Industrial Engineering and Management, The Technion, Haifa and Department of Economics and Department of Mathematics University of California, Los Angeles.

.(1993b), "Potential Games," mimeo, Faculty of Industrial Engineering and Management, The Technion, Haifa and Department of Economics and Department of Mathematics University of California, Los Angeles.

Nyarko, Y. (1991), "Learning in Mis-specified Models and the Possibility of Cycles," Journal of Economic Theory, 55: 416-427.

(1992a), "Bayesian Learning in Repeated Games Leads to Correlated Equilibria," Working Paper No. 92-26, New York University.

(1992b), "Bayesian Learning Without Common Priors and Convergence to Nash Equilibria," Working Paper No. 92-25, New York University. (1993a), "The 'Types' of a Bayesian Equilibrium," Working Paper No. 93-36, New York University. (1993b), "The Savage Bayesian Foundations of Economic Dynamics," Working Paper No. 93-35, New York University.

Pearce, D., "Rationalizable Strategic Behavior and the Problem of Perfection," Econometrica, $52: 1029-1050$

Robinson, J. (1951), "An Iterative Method of Solving a Game," Annals of Mathematics, 54:296301

Rosenthal, R.,(1973), “A Class of Games Possessing Pure-Strategy Nash Equilibria," International Journal of Game Theory, 2:65-67. 
Sanchirico, C. W. (1996), "A Probabilistic Model of Learning in Games," Columbia University, Department of Economics, Discussion Paper No. 9596-22. (1996), "A Probabilistic Model of Learning in Games," mimeo, Columbia University, forthcoming in Econometrica.

Shapley, L. S. (1964) "Some Topics in Two Person Games," Advances in Game Theory, 26:1-28. Topkis, D. M. (1978), "Equilibrium Points in Nonzero-Sum $n$-Person Submodular Games," Siam Journal of Control and Optimization, 17(6):773-87.

Vives, X., (1990), "Nash Equilibrium with Strategic Complementarities," Journal of Mathematical Economics, 19(3): 305-321. 


\section{\begin{tabular}{c|c|c|}
\multicolumn{1}{c}{$P$} & $G$ \\
\hline & $1, l$ & 0,0 \\
\hline & 0,0 & $1, I$ \\
\hline
\end{tabular}}

GAME 1 


\begin{tabular}{|c|c|c|}
\hline & $\mathbf{L}$ & $\mathbf{R}$ \\
\hline & 10,10 & $\mathbf{0 , 1 0}$ \\
\hline & 0,0 & 0,0 \\
\hline
\end{tabular}

GAME 2 


\begin{tabular}{|c|c|c|c|}
\hline & $L$ & $M$ & $R$ \\
\hline $\mathbf{T}$ & 1 & -1 & $-1 / 2$ \\
\hline & -1 & 1 & $-1 / 2$ \\
\hline & $1 / 2$ & $1 / 2$ & 0 \\
\hline
\end{tabular}

GAME 3: Augmented Matching Pennies 


\begin{tabular}{|c|c|c|c|c|}
\hline & 1 & 2 & 3 & 4 \\
\hline 1 & $1,-1$ & 111 & $0,-3$ & 0,2 \\
\hline 2 & $1 \%$ & $11-1$ & 0,2 & $0,-3$ \\
\hline 3 & 0,2 & $0,-3$ & $1,-1$ & -111 \\
\hline 4 & $0,-3$ & 0,2 & $-1,1$ & $1,-1$ \\
\hline
\end{tabular}

GAME 4 


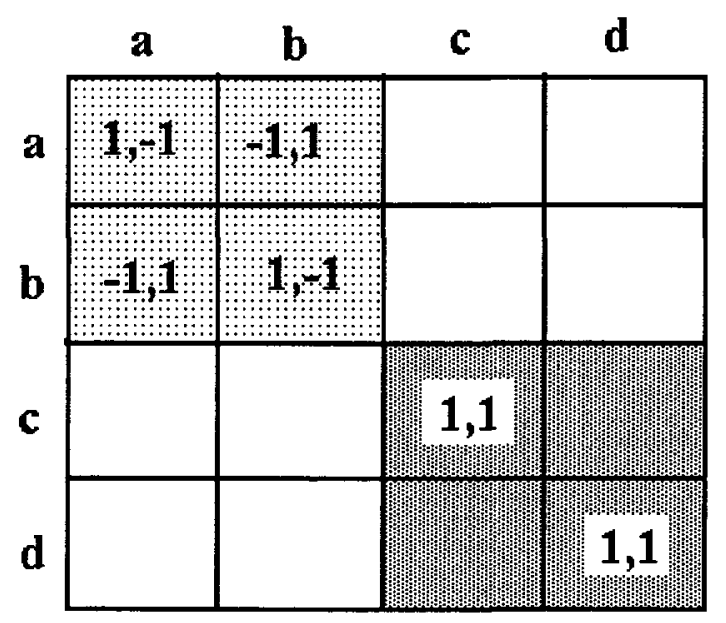

GAME 5 
'I would like to thank Luca Anderlini, Truman Bewley, Margaret Bray, Subir Bose, Joseph Chang, Jonathan Conning, John Geanakoplos, Caroline Gentile, Henry Hansmann, Al Klevorick, Sujoy Mukerji, Roger Myerson, Thomas Nyiro, Rafael Rob, Susan Rose-Ackerman, members of the Game Theory Reading Group at Yale, participants of the 1994 Summer Workshop of the Stanford Institute for Theoretical Economics (SITE), the 1994 North American Summer Meetings of the Econometric Society, and the Olin Summer Workshop at Yale Law School for their helpful comments and suggestions. I am grateful to Phil Reny for an especially useful conversation on potential games. Special thanks go to David Pearce for his advice and encouragement.

${ }^{2}$ Such research includes (but is not limited to) Crawford (1992), Foster and Young (1990), Fudenberg and Kreps (1988 and 1992), Fudenberg and Levine (1993a and b), Gul (1991), Jordan (1991b), Kalai and Lehrer (1993), Kandori, Malaith and Rob (1992), Kandori and Rob (1992a), Krishna (1991), Li Calzi (1992), Milgrom and Roberts (1991), Monderer and Shapley (1993a and b), Nyarko (1992a), Sanchirico (1993).

${ }^{3}$ The model is set in the usual repeated game framework, but the two key assumptions are stated in terms of the likelihood of beliefs and actions conditional on the history of play. The model shows how convergence of belief and action may follow from the interaction of two seemingly unrelated "forces," both of which seem present in real world interaction. The first force consists of the feedback produced when certain types of histories, "inclusive sets," are subjected to the assumption that the more often a subset of stage game profiles has been played in, the more likely it is to be "salient" in players' beliefs regarding play in the incipient stage game. A subset of action profiles is "salient" at $\mathrm{t}$ when players believe either that their opponents will play again 
in (their factors of) that subset, or that their opponents believe that their opponents will do so, or etc...up to any order. Whenever an inclusive set of actions is salient (and players are sufficiently impatient), it will in fact be played in. This will then make it more likely to be salient again in the following period--hence the feedback. The second force, "entropy," is a best response based noise assumption designed to reflect the indeterminacy of rational strategic interaction (c.f. rationalizability). The actual assumption requires that every best response to "recent" history be at least possible in the incipient stage game.

${ }^{4}$ Basu and Weibull (1990) call inclusive sets "strategy subsets closed under rational behavior" or "CURB""s for short. Minimal inclusive sets are then "minimal curbs." I chose a simpler terminology which serves to emphasize the duality between inclusive sets and "excusive sets," which are defined with the reverse inclusion. (See REMARK 1, infra.) ${ }^{5}$ Minimal inclusive sets will always be contained in the set of rationalizable profiles. ${ }^{6}$ An equilibrium is strict if deviation always leads to strictly lower payoffs. ${ }^{7}$ This proviso is not needed for games with bandwagon effects, since it is already built into the definition of the class. The other classes of games include those in which all payoffs are zero. Obviously, then, some additional genericity assumption will be needed. ${ }^{8}$ Following Bernheim (1984) and Pearce (1984). ${ }^{9}$ Roughly, a sequence of action profiles is consistent with sophisticated learning if each player always plays a best response to "recent" history, where for every time $t$ there comes a point after which it is no longer part of what is considered recent.

${ }^{10}$ In fictitious play, the probability that each player places on each of his opponents taking a given action at time $t$ is assumed equal to the proportion of times in the past that his opponent has taken 
that action. Players then play myopic best responses to these beliefs. See Fudenberg and Kreps (1992) for a helpful discussion of the properties of this learning rule.

${ }^{11}$ That is, without "ties" in the payoff matrix. Actually, it suffices that all pure equilibria are strict.

${ }^{12}$ I thank Phil Reny for bringing these papers to my attention and posing the question of how small minimal inclusive sets might be in such games.

${ }^{13}$ As usual, given any product $X_{1} \times \mathrm{K} \times X_{n}, X$ denotes the product itself, $X_{i}$ denotes the $i$ th factor, and $X_{-i}$ denotes the product of all factors except the $i$ th. Subsets of, or measures on, such products receive the same subscripting for convenient reference.

${ }^{14}$ So far, I have only defined the finite game and so this qualification is superfluous. In the next section, I consider the game's mixed extension. To avoid confusion, I will often refer to the equilibria of the finite game as "pure" equilibria. Moreover, when in subsequent sections I refer to "mixed" equilibria of the mixed extension, I will mean to include pure equilibria, as well, viewing them as "degenerate" mixed strategies.

${ }^{15}$ But note that Krishna resolves ties in fictitious play by assuming that players play the largest best response.

${ }^{16} \mathrm{~A}$ partial ordering is transitive, reflexive and antisymmetric $(x \geq y \wedge y \geq x \Rightarrow x=y$, where the equality means "same point" not the conjunction of the two conditions). The antisymmetry requirement in the definition of a partial ordering insures that we can not make every game supermodular by simply setting all profiles equal (in the sense of $x \geq y \wedge y \geq x$ ) in the ordering. ${ }^{17}$ Milgrom and Roberts (1990) also impose the condition "order continuity," This condition is vacuous in finite games. 
${ }^{18}$ That is, the relation is a partial ordering and $A_{i}$ contains the least upper bound and greated lower bound of every one of its subsets, where. e.g., the least upper bound (lub) of a subset S of a set $X$ is an element in $X$ which is "greater than" all elements in $S$ and "less than" all elements in $X$ with the same property.

19 That is, $\forall a_{i}, a_{i}^{\prime} \in A_{i}, a_{-i} \in A_{-i}, \quad \pi_{i}\left(a_{i}, a_{-i}\right)+\pi_{i}\left(a_{i}^{\prime}, a_{.}\right) \leq \pi_{i}\left(\mathrm{glb}\left\{a_{i}, a_{i}^{\prime}\right\}, a_{-i}\right)+\pi_{i}\left(\operatorname{lub}\left\{a_{i}, a_{i}^{\prime}\right\}, a_{-i}\right)$.

20 That is, $a_{i} \geq_{i} a_{i}^{\prime} \wedge a_{-i} \geq_{-i} a_{-i}^{\prime} \Rightarrow \pi_{i}\left(a_{i}, a_{-i}\right)-\pi_{i}\left(a_{i}^{\prime}, a_{-i}\right) \geq \pi_{i}\left(a_{i}, a_{-i}^{\prime}\right)-\pi_{i}\left(a_{i}^{\prime}, a_{-i}^{\prime}\right)$.

${ }^{21}$ Take any minimal inclusive set, $I$. Since $I$ is exact, it is rectangular. Suppose that each $I_{i}$ is a complete lattice in its own right. The restriction of $\mathrm{G}$ to $I$ will be a supermodular game by LEMMA 2. Milgrom and Roberts' (1990) Theorem 5 then guarantees that the largest and smallest profiles in the restricted game which survive the iterated elimination (from $I$ ) of strategies strictly dominated on point beliefs over $I$ will be pure strategy Nash equilibria. Then since all pure equilibria are strict, $I$ will contain a strict Nash equilibrium (rel. the restricted game). Since $I$ is inclusive in $\mathrm{G}$, this equilibrium will also be a strict equilibrium for $\mathrm{G}$. Hence, since $I$ is minimal in $\mathrm{G}$, it must consist solely of this equilibrium.

${ }^{22}$ This argument also shows that the marginal bandwagon property implies the "total bandwagon property" (also defined in the same paper) for undominated strategies. Cf. the assertion on p. 6 that "TBP and MBP are not nested assumptions." 


\section{5-1996 Discussion Paper Series}

Department of Economics

Columbia University

1022 International Affairs Bldg.

420 West 118th Street

New York, N.Y., 10027

The following papers are published in the 1995-96 Columbia University Discussion Paper series which runs from early November to October 31 of the following year (Academic Year).

Domestic orders for discussion papers are available for purchase at the cost of $\$ 8.00$ (U.S.) Per paper and $\$ 140.00$ (US) for the series.

Eoreign orders cost $\$ 10.00$ (US) per paper and $\$ 185.00$ for the series.

To order discussion papers, please write to the Discussion Paper Coordinator at the above address along with a check for the appropriate amount, made payable to Department of Economics, Columbia University. Please be sure to include the series number of the requested paper when you place an order. 


\section{5-96 Discussion Paper Series}

9596-01 Protectionist Response to Import Competition in Declining Industries Reconsidered

9596-02 New Estimates on Climate Demand: Evidence from Location Choice

9596-03 Enforcement by Hearing

9596-04 Preferential Trading Areas and Multilateralism: Strangers, Friends or Foes?

9596-05 Simplification, Progression and a Level Playing Field

9596-06 The Burden of Proof in Civil Litigation

9596-07 Market Structure and the Timing of Technology Adoption

9596-08 The Emergence of the World Economy

9596-09 The Global Age: From a Skeptical South to a Fearful North

9596-10 A Conformity Test for Cointegration

9596-11 Identification and Kullback Information in the GLSEM

9596-12 Informational Leverage and the Endogenous Timing of Product Introductions

9596-13 Changes in Wage Inequality

9596-14 The Design of Monte Carlo Experiments for VAR Models

9596-15 A Topological Invariant for Competitive Markets

9596-16 Topology and Invertible Maps

9596-17 Smooth Infinite Economies by: J. Choi

by: M. Cragg

M. Kahn

by: C. Sanchirico

by: J. Bhagwati

A. Panagariya

by: W. Vickrey

by: C. Sanchirico

by: J. Choi

M. Thum

by: R. Findlay

by: J. Bhagwati

by: P. Dhrymes

by: P. Dhrymes

by: J. Choi

by: J. Mincer

by: P. Dhrymes

by: G. Chichilnisky

by: G. Chichilnisky

by: G. Chichilnisky 


\section{5-96 Discussion Paper Series}

9596-18 Measuring Neighborhood Investments: Urban Quality of Life Expenditures by Race

9596-19 The Silver Lining of Industrial Decline: Rust Belt Manufacturing's Impact on Particulates

9596-20 Education's Role in Explaining Diabetic Health Investment Differentials

9596-21 Limited Arbitrage and Uniqueness of Market Equilibrium

9596-22 A Probabilistic Model of Learning in Games

9596-23 Minimal Inclusive Sets in Special Classes of Games

9596-24 'Globalization' and Vertical Structure

9596-25 Corruption, Black Markets and the Fiscal Problem in LDC's: Some Recent Findings

9596-26 Black Markets and Optimal Evadable Taxation by: D. Dipasquale M. Kahn

by: M. Kahn

by: M. Kahn

by: G. Chichilnisky

by: C. Sanchirico

by: C. Sanchirico

by: J. McLaren

by: J. McLaren

by: J. McLaren 